\title{
Communication
}

\section{Pre-pregnancy BMI but not mild stress directly influences Interleukin-6 levels and insulin sensitivity during late pregnancy}

\author{
Ilena Bauer ${ }^{1,2, *}$, Franziska Schleger ${ }^{1,2}$, Julia Hartkopf ${ }^{1,2}$, Ralf Veit ${ }^{1}$, Muriel Breuer ${ }^{1}$, \\ Nathalie Schneider ${ }^{1}$, Jan Pauluschke-Fröhlich ${ }^{3}$, Andreas Peter ${ }^{1,2,4}$, Hubert Preiss $1^{1,2,5}$, \\ Andreas Fritsche ${ }^{1,2,6}$, Louise Fritsche $e^{1,2,6}$ \\ ${ }^{1}$ Institute for Diabetes Research and Metabolic Diseases, Helmholtz Center Munich, University of Tuebingen, 72076 Tuebingen, Germany \\ ${ }^{2}$ German Center for Diabetes Research (DZD e.V.), 85764 Neuherberg, Germany \\ ${ }^{3}$ Department of Obstetrics and Gynaecology, University Hospital Tuebingen, 72076 Tuebingen, Germany \\ ${ }^{4}$ Department for Diagnostic Laboratory Medicine, Institute for Clinical Chemistry and Pathobiochemistry, University Hospital of Tübingen, 72076 \\ Tuebingen, Germany \\ ${ }^{5}$ Department of Pharmacy and Biochemistry, Institute of Pharmaceutical Sciences, Interfaculty Centre for Pharmacogenomics and Pharma Research, \\ University of Tuebingen, 72076 Tuebingen, Germany \\ ${ }^{6}$ Department of Internal Medicine IV, Division of Endocrinology, Diabetology, and Nephrology, University Hospital Tuebingen, 72076 Tuebingen, \\ Germany \\ *Correspondence: ilena.bauer@uni-tuebingen.de (Ilena Bauer) \\ Academic Editor: Marina Ivanišević \\ Submitted: 9 November 2021 Revised: 17 December 2021 Accepted: 23 December 2021 Published: 12 February 2022
}

\begin{abstract}
Background: This study investigates the influence of maternal stress during pregnancy on maternal insulin sensitivity and Interleukin-6 (IL-6) levels in pregnant women $(\mathrm{N}=277)$ in dependence of pre-pregnancy Body-Mass-Index (BMI). Methods: Gestational diabetes was diagnosed in 80 women. We used the Patient Health Questionnaire (PHQ-D) to investigate maternal stress during pregnancy with a higher scoring indicating higher maternal stress level. IL-6 and cortisol were measured and maternal insulin sensitivity was assessed with the non-esterified fatty acid insulin sensitivity index (NEFA-ISI). Generalized Linear Model analysis was used to analyze effects within different stress groups. Results: Maternal low stress symptoms during pregnancy showed no significant association with maternal insulin sensitivity or IL-6. Higher cortisol levels during pregnancy were associated with elevated IL-6 concentrations. Pre-pregnancy BMI had the strongest positive effect on IL-6 levels and was negatively associated with insulin sensitivity during pregnancy. Conclusions: Therefore, preconceptional interventions to reduce BMI are needed to improve maternal metabolism during pregnancy.
\end{abstract}

Keywords: Gestational diabetes mellitus; Maternal stress; Obesity; Interleukin-6

\section{Introduction}

The cytokine Interleukin-6 (IL-6) has important functions in immune response, hematopoiesis, and inflammation [1]. Together with other components of the immune system, IL-6 plays an important role in maintaining pregnancy by controlling the inflammatory environment [2]. Increased IL-6 levels can have far-reaching effects on pregnancy outcome and increase potential risk factors for disease later in life [3].

Not only inflammation but also maternal stress is a risk factor for elevated IL-6 levels during pregnancy [46]. Previous studies demonstrated an association between maternal stress and metabolism of the offspring including increased risk of obesity in childhood and adulthood [7]. Even in non-pregnant subjects, a significant correlation between BMI and IL-6 levels were reported [8].

Several studies showed evidence that psychosocial stress increases IL-6 and fetal and maternal cortisol in late pregnancy and reduces maternal insulin sensitivity [9-11]. During late gestation, the activity of the fetal stress sys- tem is enhanced and the placenta is considered as a stresssensitive organ. In case of an adverse intrauterine environment, such as inflammation, a premature activation of this system may occur [11]. A recent report has shown that IL-6/cortisol levels are also measurable in cord blood [12]. But the placenta also has protective properties. These are mainly regulated by $11 \beta$-hydroxysteroid dehydrogenase type 1 which controls fetal exposure to glucocorticoids like cortisol [12]. In non-pregnant subjects, a correlation between cortisol levels, IL-6 and BMI was reported [13]. With regard to high stress, it is well known that maternal stress levels have an impact on cortisol levels [14] and are associated with the womens' BMI during pregnancy [15].

The present study aimed to decipher the interplay between the pre-pregnancy BMI, maternal stress, IL-6 and maternal insulin sensitivity during late pregnancy in a large sample of deeply phenotyped pregnant women including gestational diabetes.

Our hypotheses were:

(1) Elevated stress levels during pregnancy are asso- 
ciated with an increase in maternal IL-6 in dependence of pre-pregnancy BMI.

(2) Elevated stress levels during pregnancy are associated with a decrease in maternal insulin sensitivity in dependence of pre-pregnancy BMI.

(3) Increased IL-6 values are associated with a reduced maternal insulin sensitivity.

In an exploratory approach, we aim to investigate the relation between cortisol and IL-6 as well as cortisol and insulin sensitivity.

\section{Materials and methods}

\subsection{Study population}

We analyzed data of the ongoing German Gestational Diabetes Study (PREG study) (Clinical trials identifier: NCT04270578), which investigates metabolic disturbances during pregnancy between gestational week 24th-31st + 6th. By in-depth phenotyping of the mother and her offspring, we aim to elucidate the relationship of maternal hyperglycemia during pregnancy and adequate treatment and its impact on the long-term health for both, mother and offspring. Women are examined during pregnancy as well as $1,2,5$ and 10 years after delivery. In this analysis, we analyzed data from pregnant participants. The study sample included 277 women, 80 with gestational diabetes (GDM).

\subsection{Maternal psychological parameter}

To assess stress we used the validated German translation of Patient Health Questionnaire (PHQ-D) [16]. The women completed the questionnaire mainly with paper pencil during the study visit or at home afterwards. The questionnaire consists of 78 items in total. Diagnostic criteria for major depression and other depressive disorders, panic disorder and other anxiety disorders, bulimia nervosa, bingeeating disorder, somatoform disorder and alcohol abuse are asked. There is a two to five-level response scale for each question.

Within the PHQ-D there are both categorical and continuous variables. A dimensional evaluation is possible for the somatization module ("PHQ-15"), the depression module ("PHQ-9") and the stress module ("Schweregrad Stress"). For the analysis we explicitely used the "Stress" subscale to determine the stress level of the women. The subscale "Stress" contains ten items with the rating 0 ("not impaired"), 1 ("slightly impaired") or 2 ("severely impaired"). In the "Stress" subscale, the result is a sum of scales between 0 (no stress) to 20 (high stress).

\subsection{Maternal physiological parameters}

In all participants, a 5-point oral glucose tolerance test (OGTT) with $75 \mathrm{~g}$ glucose was performed in the morning after an overnight fast. GDM was diagnosed following the national guidelines of the German Diabetes Association (DDG) [17] in accordance with the International Association of Diabetes in Pregnancy Study Groups (IADPSG) [18]. Women without gestational diabetes diagnosis were defined as women with normal glucose tolerance (NGT).

Maternal insulin sensitivity was calculated with the non-esterified-free-fatty-acids insulin sensitivity index (NEFA-ISI) [19].

Pre-gestational weight and height was taken from maternal medical logs and the pre-pregnancy BMI (preBMI) was calculated weight $(\mathrm{kg}) / \mathrm{height}^{2}\left(\mathrm{~m}^{2}\right)$. Women were assigned to three groups, depending on their preBMI: women with normal weight (BMI 18.5-24.9; $\mathrm{N}=179$ ), overweight (BMI 25.0-29.9; $\mathrm{N}=44$ ), and obesity (BMI $\geq 30.0 ; \mathrm{N}=72$ ) (see Supplementary Table 1).

Maternal IL-6 concentrations (ng/L) were analyzed with High Sensitive Enzyme Linked Immunosobent assay (ELISA) (R\&D systems, Minneapolis, MN, USA; assay range: $0-10 \mathrm{pg} / \mathrm{mL}$, minimum detectable dose: 0.031 $\mathrm{pg} / \mathrm{mL}$ ) according to the manufacturer's instructions. Cortisol levels were analyzed on an automated ADVIA Centaur XPT immunoassay system (Siemens Healthineers, Eschborn, Germany, assay range: 5.6-2100 nmol/L).

\subsection{Statistical analysis}

All statistical analyses were performed with IBM SPSS Statistics software version 26 (IBM Corp., Armonk, NY, USA). Outliers were identified using three standard deviations as cutoff. We used a Generalized Linear Model (GzLM) with gamma as distribution and log as canonical link function. GzLM was used to test effects of maternal stress on IL-6 and effects of IL-6 on maternal insulin sensitivity. To determine the goodness of fit of the different statistical models and to prevent overfitting, we used the Akaike-Information-Criterium (AIC). For testing the effects in the model, a Wald-Chi-Square test with a significance level at $p<0.05$ was used. In case of significant model effects, pairwise comparisons for factors and interactions were computed and parameter estimates are reported. To account for multiple comparisons, BonferroniHolm correction was applied. Missing values in the PHQD questionnaire were handled as described in Kocalevent $e t$ al. [20]. PreBMI, GDM status, gestational age (in weeks) (GA) and cortisol level were independent variables in the model. Two-way interactions between stress and the other variables were computed. To determine relations between cortisol and stress or IL-6, we performed a Pearson's correlation (in case of normally distributed data) or Spearman correlation (in case of non-normally distributed data).

\section{Results}

\subsection{Population and procedure}

The main sample included 277 women, 80 with GDM. Detailed characteristics are displayed in Table 1. Overall, we observed a right skewed distribution of the stress levels, indicating that the majority of subjects were only moderately/mildly stressed. According to the study of Braig et al. [21] we divided the subjects based on their stress scores in three groups: no stress (ratings 0 and 1), low stress (ratings 
Table 1. Detailed anthropometric and metabolic characteristics for GDM and NGT women (in total, $\mathbf{N}=277$ ).

\begin{tabular}{lccc}
\hline Parameter & NGT & GDM & $p$-value \\
\hline $\mathrm{N}$ & 197 & 80 & \\
$\mathrm{AGE}($ years $)$ & $31.85(4.68)$ & $33.12(4.82)$ & 0.043 \\
BMI $\left(\mathrm{kg} / \mathrm{m}^{2}\right)$ & $26.22[23.43,29.76]$ & $28.86[25.75,32.61]$ & 0.001 \\
Pre-pregnancy BMI $\left(\mathrm{kg} / \mathrm{m}^{2}\right)$ & $23.19[21.60,26.85]$ & $25.84[22.78,30.80]$ & 0.001 \\
Gestational Age (weeks) & $26.90(2.01)$ & $26.74(1.76)$ & 0.519 \\
NEFA-ISI & $3.44[2.66,4.38]$ & $2.35[1.89,3.40]$ & $<0.001$ \\
Parity (\%) & & & 0.042 \\
$\quad$ multiparous & $85(43.1)$ & $46(57.5)$ & 0.686 \\
$\quad$ nulliparous & $112(56.9)$ & $34(42.5)$ & 0.002 \\
Cortisol (nmol/L) & $721.00[622.00,804.00]$ & $705.50[617.75,859.00]$ & 0.437 \\
IL-6 (ng/L) & $0.91[0.56,1.34]$ & $1.19[0.78,1.73]$ & \\
PHQ-D Subscale: & & & 0.643 \\
$\quad$ Severity of stress (Schweregrad Stress) & $3.00(2.75)$ & $3.29(2.89)$ & \\
Stress groups & & $29(36.2)$ & \\
$\quad$ mild stress & $69(35.0)$ & $29(36.2)$ & $22(27.5)$ \\
$\quad$ low stress & $63(32.0)$ & $65(33.0)$ & \\
$\quad$ no stress &
\end{tabular}

Data are presented as means (SD), median [IQR] and counts (\%). IQR, interquartilerange; SD, standard deviation; N, sample size; GDM, women with gestational diabetes; NGT, women with normal glucose tolerance.

2 and 3 ) and mild stress (ratings $\geq 4$ ).

\subsection{Maternal stress is not associated with IL-6 levels during pregnancy}

The GzLM revealed no significant main effect of maternal stress on IL-6 (Fig. 1). However, a main effect for preBMI on IL-6 was observed $\left(\chi^{2}=36.64, p<0.001\right)$. With increasing preBMI, women had significant higher IL6 values. We found higher IL-6 in GDM compared to women with normal glucose tolerance (NGT) $\left(\chi^{2}=5.25\right.$ $p=0.022$, beta $=0.181)$. In an explorative approach, we found a significant positive correlation of cortisol with IL$6\left(\chi^{2}=6.21 p=0.013\right.$, beta $\left.=0.001\right)($ Table 2$)$.

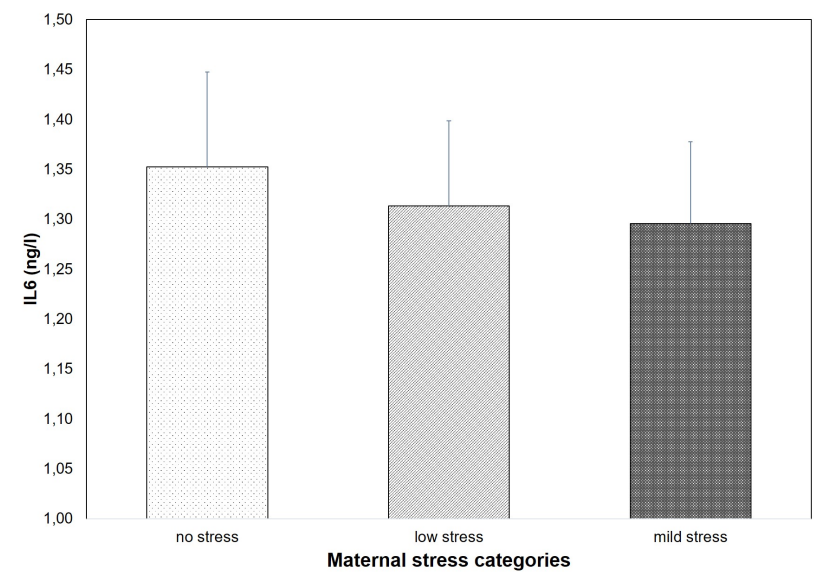

Fig. 1. Maternal stress categories and Interleukin-6 (IL-6) levels. no stress: $\mathrm{N}=76$; low stress: $\mathrm{N}=87$; mild stress: $\mathrm{N}=97$.
Table 2. Effect of maternal stress on Interleukin-6, complete model of GzLM analysis.

\begin{tabular}{lcc}
\hline Predictor & $\chi^{2}$ & $p$-value \\
\hline Maternal stress & & n.s. \\
PreBMI & 36.64 & $p<0.001$ \\
Group (GDM/NGT) & 5.25 & $p=0.022$ \\
IL-6 & 6.21 & $p=0.013$ \\
\hline
\end{tabular}

GDM, Women with gestational diabetes; NGT, Women with normal glucose tolerance; IL-6, Interleukin-6; PreBMI, pre-pregnancy BMI; IS, insulin sensitivity; n.s., not significant.

We analyzed the effect of cortisol on maternal stress and found no effect in the GzLM analysis. With increasing IL-6, women had significant higher cortisol levels. We observed no significant main effect for group (GDM/NGT) or preBMI.

\subsection{Maternal stress is not associated with insulin} sensitivity

We found no significant effect of maternal stress on insulin sensitivity. We found a significant main effect of preBMI on maternal insulin sensitivity $\left(\chi^{2}=97.76, p<\right.$ 0.001). With increasing BMI, women had significant lower insulin sensitivity. We observed a significant main effect for group (GDM/NGT), with increased insulin sensitivity in NGT opposed to GDM women $\left(\chi^{2}=20.28, p=0.001\right)$. IL6 values were negatively associated with insulin sensitivity $\left(\chi^{2}=16.53, p<0.001\right.$, beta $\left.=-0.106\right)($ Table 3$)$. 
Table 3. Effect of maternal stress on insulin sensitivity, complete model of GzLM analysis.

\begin{tabular}{lcc}
\hline Predictor & $\chi^{2}$ & $p$-value \\
\hline Maternal stress & & n.s. \\
PreBMI & 97.76 & $p<0.001$ \\
Group (GDM/NGT) & 20.28 & $p=0.001$ \\
IL-6 & 16.53 & $p<0.001$ \\
\hline
\end{tabular}

GDM, Women with gestational diabetes; NGT, Women with normal glucose tolerance; IL-6, Interleukin-6; PreBMI, pre-pregnancy BMI; IS, insulin sensitivity; n.s., not significant.

\section{Discussion}

In our study population, maternal low stress symptoms during pregnancy were not associated with maternal insulin sensitivity or IL-6, independent of presence or absence of gestational diabetes. Women with pre-pregnancy overweight or obesity showed higher IL-6 and lower insulin sensitivity compared to women with normal weight. These effects are also independent of the presence of GDM. Unexpectedly, the degree of maternal stress was not associated with IL-6. This might be explained by the rather low stress in our cohort (scores not higher than 13 on a scale between 0 and 20). It is well-known that decreased insulin sensitivity is the key pathophysiological mechanism underlying the development of gestational diabetes [22] and that maternal IL-6 levels are increased in pregnancies with GDM [23]. Besides, a study reported an increased IL-6 concentration by obesity independently of insulin resistance status [24]. A study by Cousson-Read [9], demonstrated a significant association between prenatal stress and the cytokine profile in pregnant women across pregnancy. Valsamakis and colleagues [11] reported a negative correlation between prenatal anxiety symptoms and maternal insulin sensitivity in eighty-two pregnant women but did not report any correlation between prenatal stress and IL-6. Our study shows that pre-pregnancy BMI is associated with maternal insulin sensitivity and IL-6 levels in the third trimester of pregnancy. IL-6 is secreted from adipose tissue $[25,26]$ and obesity is recognized as a state of subclinical inflammation, which contributes to insulin resistance. An association of adiposity and proinflammatory cytokines, including IL-6, was shown during pregnancy [27] arguing for a direct effect of BMI on IL-6 levels.

One limitation of our study is that we used a questionnaire which asked about the general stress level. Subtle or other minor stress symptoms and pregnancy-related questions were not explicitly queried.

\section{Conclusions}

The mechanisms between IL-6 levels and maternal psychosocial stress and the direction of causality is not yet fully understood and needs to be further investigated. This is necessary to provide women with optimal care during pregnancy or even in the preconception phase with regard to the pre-pregnancy BMI and stress management programs.

\begin{abstract}
Abbreviations
BMI, Body-Mass-Index; DSM-V, Diagnostic and Statistical Manual of Mental Disorders; GA, Gestational age; GDM, Gestational diabetes mellitus; GzLM, Generalized Linear Model; IL-6, Interleukin-6; NEFAISI, Nonesterified-free-fatty-acids insulin sensitivity index; NGT, Normal glucose tolerance; OGTT, Oral Glucose Tolerance Test; PHQ-D, Patient Health Questionnaire (German translation); preBMI, Pre-pregnancy BMI.
\end{abstract}

\section{Author contributions}

IB analyzed and interpreted the data and drafted the manuscript. JH, FS, NS, MB and JP researched data and FS contributed to interpretation of the data. AF and RV contributed to analysis and interpretation of the data. AP supervised the laboratory measurements and interpreted results. LF and HP were the study supervisors and contributed to analysis and interpretation of the data. All authors contributed to the final manuscript and read and approved the final manuscript.

\section{Ethics approval and consent to participate}

Written informed consent was received from participants prior to all measurements. The local ethics committee approved the protocol and the study was conducted in accordance with the declaration of Helsinki.

Clinical Trial registration: We analyzed data of the ongoing PREG study [28] (Clinical trials identifier: NCT04270578).

\section{Acknowledgment}

The authors acknowledge the cooperation of all women taking part voluntarily in this study. We also thank Magdalene Weiss for her substantial support during the study and Dorothee Neuscheler for their excellent technical assistance. Additionally, we would like to thank Ellen Kern and Robert Wagner for their support.

\section{Funding}

This research received no external funding.

\section{Conflict of interest}

The authors declare no conflict of interest.

\section{Supplementary material}

Supplementary material associated with this article can be found, in the online version, at https://www.imrpre ss.com/journal/FBL/27/2/10.31083/j.fbl2702056. 


\section{References}

[1] Jones SA. Directing transition from innate to acquired immunity: defining a role for IL-6. Journal of Immunology. 2005; 175: 3463-3468.

[2] Munoz-Suano A, Hamilton AB, Betz AG. Gimme shelter: the immune system during pregnancy. Immunological Reviews. 2011; 241: 20-38.

[3] Prins JR, Gomez-Lopez N, Robertson SA. Interleukin-6 in pregnancy and gestational disorders. Journal of Reproductive Immunology. 2012; 95: 1-14.

[4] Finy MS, Christian LM. Pathways linking childhood abuse history and current socioeconomic status to inflammation during pregnancy. Brain, Behavior, and Immunity. 2018; 74: 231-240.

[5] Dipietro JA. Maternal stress in pregnancy: considerations for fetal development. The Journal of Adolescent Health. 2012; 51: S3-S8.

[6] Kinsella MT, Monk C. Impact of Maternal Stress, Depression and Anxiety on Fetal Neurobehavioral Development. Clinical Obstetrics \& Gynecology. 2009; 52: 425-440.

[7] Entringer S. Impact of stress and stress physiology during pregnancy on child metabolic function and obesity risk. Current Opinion in Clinical Nutrition and Metabolic Care. 2013; 16: 320-327.

[8] Patsalos O, Dalton B, Himmerich H. Effects of IL-6 Signaling Pathway Inhibition on Weight and BMI: A Systematic Review and Meta-Analysis. International Journal of Molecular Sciences. 2020; 21: 6290.

[9] Coussons-Read ME, Okun ML, Nettles CD. Psychosocial stress increases inflammatory markers and alters cytokine production across pregnancy. Brain, Behavior, and Immunity. 2007; 21: 343-350.

[10] Leff Gelman P, Mancilla-Herrera I, Flores-Ramos M, Saravia Takashima MF, Cruz Coronel FM, Cruz Fuentes C, et al. The cytokine profile of women with severe anxiety and depression during pregnancy. BMC Psychiatry. 2019; 19: 104.

[11] Valsamakis G, Papatheodorou DC, Chalarakis N, Vrachnis N, Sidiropoulou EJ, Manolikaki M, et al. In pregnancy increased maternal STAI trait stress score shows decreased insulin sensitivity and increased stress hormones. Psychoneuroendocrinology. 2017; 84: 11-16.

[12] Dahlerup BR, Egsmose EL, Siersma V, Mortensen EL, Hedegaard M, Knudsen LE, et al. Maternal stress and placental function, a study using questionnaires and biomarkers at birth. PLoS ONE. 2019; 13: e0207184.

[13] van der Valk ES, van der Voorn B, Iyer AM, Mohseni M, Leenen PJM, Dik WA, et al. Hair cortisol, obesity and the immune system: Results from a 3 year longitudinal study. Psychoneuroendocrinology. 2021; 134: 105422.

[14] Urizar GG, Milazzo M, Le H, Delucchi K, Sotelo R, Muñoz RF. Impact of stress reduction instructions on stress and cortisol levels during pregnancy. Biological Psychology. 2004; 67: 275282 .
[15] Aubuchon-Endsley NL, Bublitz MH, Stroud LR. Pre-pregnancy obesity and maternal circadian cortisol regulation: Moderation by gestational weight gain. Biological Psychology. 2014; 102: 38-43.

[16] Gräfe K, Zipfel S, Herzog W, Löwe B. Screening psychischer Störungen mit dem "Gesundheitsfragebogen für Patienten (PHQ-D)". Diagnostica. 2004; 50: 171-181.

[17] Ute Schäfer-Graf. Gestationsdiabetes mellitus (GDM), Diagnostik, Therapie und Nachsorge: praxisempehlung - Kurzfassung der S3-Leitlinie (AWMF-Registernummer: 057-008). Diabetologie und Stoffwechsel. 2019; 14: 196-206.

[18] Duran A. Introduction of IADPSG Criteria for the Screening and Diagnosis of Gestational Diabetes Mellitus Results in Improved Pregnancy Outcomes at a Lower Cost in a Large Cohort of Pregnant Women: The St. Carlos Gestational Diabetes Study. Diabetes Care. 2014; 37: 2442-2450.

[19] Wagner R, Fritsche L, Heni M, Fehlert E, Stefan N, Staiger H, et al. A novel insulin sensitivity index particularly suitable to measure insulin sensitivity during gestation. Acta Diabetologica. 2016; 53: 1037-1044.

[20] Kocalevent R, Hinz A, Brähler E. Standardization of a screening instrument (PHQ-15) for somatization syndromes in the general population. BMC Psychiatry. 2013; 13: 91.

[21] Braig S, Logan CA, Reister F, Rothenbacher D, Genuneit J. Psychosocial stress and longitudinally measured gestational weight gain throughout pregnancy: the Ulm SPATZ Health Study. Scientific Reports. 2020; 10: 1996.

[22] Catalano PM. Trying to understand gestational diabetes. Diabetic Medicine: a Journal of the British Diabetic Association. 2014; 31: 273-281.

[23] Hassiakos D, Eleftheriades M, Papastefanou I, Lambrinoudaki I, Kappou D, Lavranos D, et al. Increased Maternal Serum Interleukin-6 Concentrations at 11 to 14 Weeks of Gestation in Low Risk Pregnancies Complicated with Gestational Diabetes Mellitus: Development of a Prediction Model. Hormone and Metabolic Research. 2016; 48: 35-41.

[24] Bastard J, Maachi M, Lagathu C, Kim MJ, Caron M, Vidal $\mathrm{H}$, et al. Recent advances in the relationship between obesity, inflammation, and insulin resistance. European Cytokine Network. 2006; 17: 4-12.

[25] Hoene M, Weigert C. The role of interleukin-6 in insulin resistance, body fat distribution and energy balance. Obesity Reviews. 2008; 9: 20-29.

[26] Pendeloski KPT, Ono E, Torloni MR, Mattar R, Daher S. Maternal obesity and inflammatory mediators: a controversial association. American Journal of Reproductive Immunology. 2017; 77: e12674.

[27] Friis CM, Paasche Roland MC, Godang K, Ueland T, Tanbo T, Bollerslev $\mathrm{J}$, et al. Adiposity-related inflammation: Effects of pregnancy. Obesity. 2013; 21: E124-E130.

[28] Fritsche L, Hummel J, Wagner R, Loeffler D, Hartkopf J, Machann J, et al. The German Gestational Diabetes Study (PREG): Rationale, Methodology and Design. 2021. (in press) 